\title{
Optimum process parameters for efficient and quality thin wall machining using firefly algorithm
}

\author{
Akash Dutta, Argha Das and \\ Shrikrishna N. Joshi* \\ Department of Mechanical Engineering, \\ Indian Institute of Technology Guwahati, \\ Guwahati, 781039, Assam, India \\ Email: akash.dutta@iitg.ernet.in \\ Email: arg@iitg.ernet.in \\ Email: snj@iitg.ernet.in \\ *Corresponding author
}

\begin{abstract}
The manufacturing requirements of the aerospace industry makes it imperative to use thin wall machining techniques to machine parts that would otherwise have to be assembled from a number of parts. To achieve high productivity, there must be increase in material removal rate, which is constrained by the geometrical accuracy and surface finish requirements. Thus, a compromise must be made between productivity and product quality. This paper presents an optimisation scheme to improve the productivity while keeping the surface finish within acceptable limits during thin-wall machining operations. Initially full factorial experiments were carried out on machining of closed thin walled pocket by varying feed, cutting speed and tool diameter. Surface roughness and material removal values for all experiments were recorded. Analysis of variance was carried out to find out the most significant process parameter. Later firefly algorithm, a nature inspired swarm optimisation technique was employed to obtain the optimum process parameters for desired performance. A confirmation experiment was carried out which indicates an error of $1.27 \%$ and $1.03 \%$ between predicted and experimental results of surface roughness and material removal rate respectively.
\end{abstract}

Keywords: thin wall machining; surface roughness; material removal rate; MRR; analysis of variance; ANOVA; interaction; optimisation; firefly algorithm.

Reference to this paper should be made as follows: Dutta, A., Das, A. and Joshi, S.N. (2017) 'Optimum process parameters for efficient and quality thin wall machining using firefly algorithm', Int. J. Additive and Subtractive Materials Manufacturing, Vol. 1, No. 1, pp.3-22.

Biographical notes: Akash Dutta is currently a PhD student in the Department of Mechanical Engineering, Indian Institute of Technology Guwahati. His research interests include additive manufacturing, CAD, CAM, soft computing techniques and optimisation. He received his Master of Technology degree in Computer Assisted Manufacturing from Indian Institute of Technology Guwahati in 2015 and his Bachelor of Engineering degree from Jorhat Engineering College in 2013. 
Argha Das is a Junior Research Fellow in the Department of Mechanical Engineering, Indian Institute of Technology Guwahati. He obtained his BTech and MTech degrees from National Institute of Technology Agartala. His research interests include machining, optimisation and computer numerical control technology.

Shrikrishna N. Joshi has completed his Doctoral studies in the area of Computer-Aided Manufacturing from IIT Bombay in the year 2009. Since then, he is working as an Assistant Professor in the Department of Mechanical Engineering, IIT Guwahati. His research interests are thin-wall machining; single point diamond turning; laser-based manufacturing; computer aided design and manufacturing (CAD/CAM); manufacturing process modelling and optimisation; and mechatronics. He has about 30 papers published in journals and conferences of national/international repute and four book chapters in reputed books.

\section{Introduction}

The dawn of the twentieth century saw a major transformation in the transportation sector with the invention of the airplane. The ever increasing demand for more convenient, cheaper and faster means of transportation together with the quest for exploration of the outer space has led to a tremendous growth of the aerospace industry. To cope up with the increasing demands for quality and quantity the manufacturers have switched to thin wall machining for those parts which were assembled from a number of other parts. The use of thin wall machining reduces the part cycle time by creating one piece flow of monolithic parts. These parts can be machined from a single block of metal which eliminates the need to manufacture multiple parts for assembling into a single one (Thin Wall Machining, 2015). Besides, these parts have excellent strength to weight ratio and cost advantages over conventional monolithic parts (Shamsuddin et al., 2013). However, to achieve low part cycle time, there must be increase in the MRR. But care should also be taken such that, while increasing the MRR surface quality does not degrade steeply. Thin wall machining finds application in automotive parts, electrodes for electro discharge machining and moulds (Kennedy and Earls, 2007). In applications like mould making and electrodes, surface finish plays a very crucial role in deciding the final usability of the end product. Thin wall machining is a special case of machining where the wall thickness is less in comparison to the other dimensions of the part. Yang (1980) has defined thin walled parts as those which satisfy the following criteria:

$$
\frac{1}{100}<\frac{t}{h}<\frac{1}{5}
$$

where $t$ is the thickness of the thin wall and $h$ is the length of the shortest edge of the wall.

Review of literature reveals a significant work on experimental as well as numerical fronts in the area of conventional milling process. Hayajneh et al. (2007) studied the effect of spindle speed, cutting feed rate and depth of cut and their interaction on surface 
roughness (SR) of end milling of aluminium by analysis of variance (ANOVA) and developed a mathematical model with multiple regression analysis to predict the SR. Wang et al. (2005) analysed the SR behaviour of micro-end milling of brass with respect to tool diameter, feed rate, depth of cut and spindle speed. They deployed ANOVA and RSM method to find out the influence level of the cutting parameters and their interaction on SR. Subsequently a quadratic mathematical model was developed by them to describe the relation between the cutting parameters and SR. Seguy et al. (2008) carried out finite element analysis for thin wall milling of 2017 aluminum alloy and developed stability and dynamic model to give detailed insight into the thin wall milling process. Michalik et al. (2014) studied the effect of up milling and down milling on SR $\left(\mathrm{R}_{\mathrm{z}}\right)$ of $\mathrm{C} 45$ steel during thin wall milling and correlates the input factors with SR. Hossain and Ahmad (2012) developed an Adaptive Network-based Fuzzy Interface System (ANFIS) to predict the SR of three dimensional end milling of aluminium with respect to cutter axis inclination angle, spindle speed, feed rate, radial depth of cut, axial depth of cut. The predicted results were then compared with ANN and RSM-based prediction results and experimental results. Thus it can be inferred that there has been a number of studies on the effect of process parameters on SR for normal machining operations.

During thin wall machining operation, at high MRRs, static and dynamic problems, particularly the self-excited vibration called chatter, appear due to the lack of stiffness of the thin walls and webs. In end milling process, the thickness of the plates is reduced gradually, which makes it even more difficult to control the accuracy of machining. The end milling of such plates is complicated, where periodically varying milling forces excite the flexible plate structures both statically and dynamically and leading to significant deformations and poor surface finish. However, a very limited information is available on the optimum process parameters that can be employed during thin wall machining process to obtain the desired process performance.

In this paper an attempt has been made to optimise the process parameters so that there is considerable increase in the MRR without compromising on surface quality.

Figure 1 The experimental setup (see online version for colours)

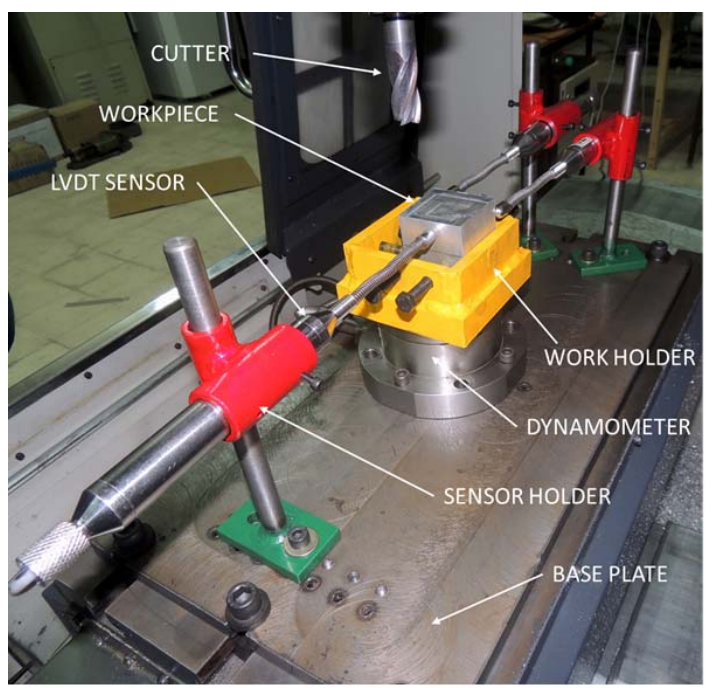


Figure 2 Initial and final specimen geometry (see online version for colours)
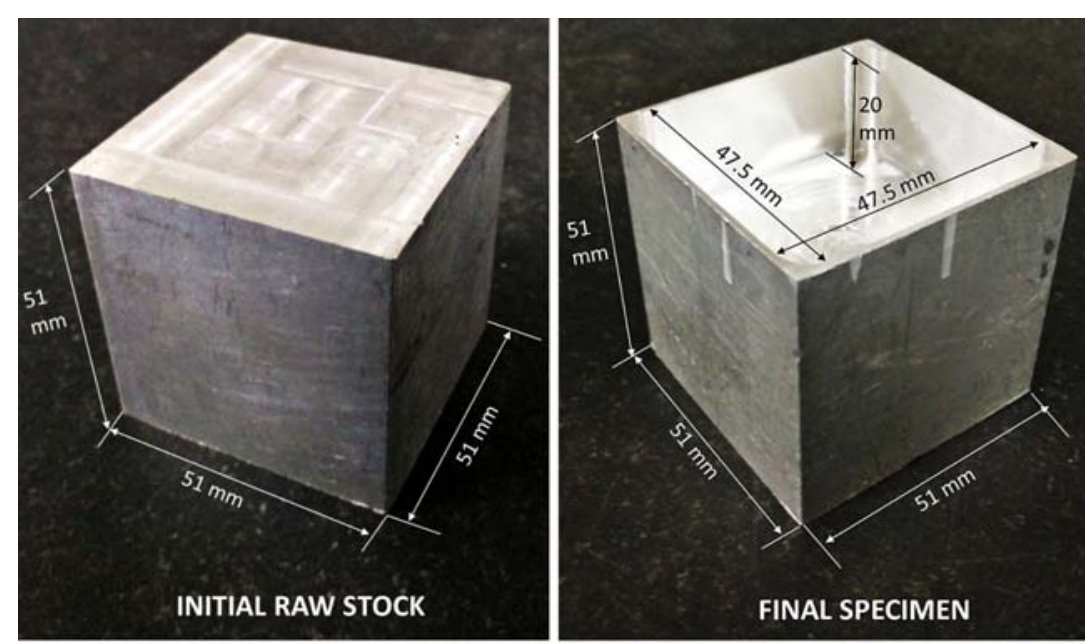

\section{Methodology}

\subsection{The experimental setup}

The experiments were carried out on a 3 -axis vertical milling machine with a maximum spindle speed of 10,000 rpm using a custom made setup shown in Figure 1. The specimen used for the experiments were made up of commercial 1000 series aluminium. A total of 27 specimens were prepared from an initial raw stock of size $51 \mathrm{~mm} \times 51 \mathrm{~mm} \times 51 \mathrm{~mm}$. The material was removed from the raw stock by end milling operation. The SR was measured by means of a contact probe type SR tester of Mitutoyo SJ-400 make. The initial and final specimen shape and size are shown in Figure 2.

\subsection{Design of experiments}

It was intended to find out the effect of the process parameters on surface finish during thin wall machining of the specimens. Besides, it was also desired that the interaction among the process parameters is also determined. It was, therefore imperative that the experiments be designed in such a way so as to accomplish all the desired goals. There are a number of approaches in which the experiments can be designed. Taguchi approach to design of experiments allows the experimenter to include a number of factors in the experiments while keeping the number of experiments to a bare minimum. For example, to design an experiment involving three factors each with three levels, the factorial design requires $3^{3}=27$ experiments to be done. However, the same can be achieved with only nine experiments using Taguchi design of experiments. But Taguchi approach is not effective in determining the interaction among the process parameters. In fact, factorial experiments are the only way to discover interactions between variables (Montgomery, 2014). Therefore, in this work, factorial design was used for the experiments with the process parameters, listed in Table 1, with a total of 27 experiments. 
Table 1 Experimental process parameters

\begin{tabular}{llccc}
\hline \multirow{2}{*}{ Process parameter } & & \multicolumn{3}{c}{ Values } \\
\cline { 3 - 5 } & & Level 1 & Level 2 & Level 3 \\
\hline Speed & $\mathrm{rpm}$ & 3,000 & 3,500 & 4,000 \\
Feed & $\mathrm{mm} /$ tooth & 0.06 & 0.08 & 0.10 \\
Tool diameter & $\mathrm{mm}$ & 8 & 10 & 12 \\
Radial depth of cut & $\mathrm{mm}$ & & 0.55 (constant) \\
Axial depth of cut & $\mathrm{mm}$ & \multicolumn{3}{c}{0.25 (constant) } \\
Helix angle & & High speed steel \\
Tool material & & \multicolumn{3}{c}{ - } \\
Number of flutes & & \multicolumn{3}{c}{} \\
\hline
\end{tabular}

Table 2 Experimental data

\begin{tabular}{|c|c|c|c|c|c|}
\hline Exp. по. & Speed rpm & Feed $\mathrm{mm} /$ tooth & $\begin{array}{c}\text { Tool diameter } \\
\mathrm{mm}\end{array}$ & $\begin{array}{c}\text { Surface } \\
\text { roughness } \mu m\end{array}$ & $\begin{array}{l}\text { MRR cu } \\
\mathrm{mm} / \mathrm{min}\end{array}$ \\
\hline 1 & 3,000 & 0.06 & 8 & 1.915 & $1,374.7$ \\
\hline 2 & 3,000 & 0.08 & 8 & 2.133 & $1,807.4$ \\
\hline 3 & 3,500 & 0.06 & 8 & 1.509 & $1,592.5$ \\
\hline 4 & 4,000 & 0.1 & 8 & 1.822 & $2,904.6$ \\
\hline 5 & 4,000 & 0.06 & 8 & 1.427 & $1,807.4$ \\
\hline 6 & 3,500 & 0.1 & 8 & 1.760 & $2,570.2$ \\
\hline 7 & 4,000 & 0.08 & 8 & 1.847 & $2,366.0$ \\
\hline 8 & 3,500 & 0.08 & 8 & 2.035 & $2,089.2$ \\
\hline 9 & 3,000 & 0.1 & 8 & 1.731 & $2,228.2$ \\
\hline 10 & 4,000 & 0.1 & 10 & 0.529 & $3,014.3$ \\
\hline 11 & 3,500 & 0.08 & 10 & 0.555 & $2,169.8$ \\
\hline 12 & 3,000 & 0.06 & 10 & 0.491 & $1,428.6$ \\
\hline 13 & 4,000 & 0.06 & 10 & 0.517 & $1,877.5$ \\
\hline 14 & 3,500 & 0.06 & 10 & 0.513 & $1,654.7$ \\
\hline 15 & 3,000 & 0.08 & 10 & 0.589 & $1,877.5$ \\
\hline 16 & 4,000 & 0.08 & 10 & 0.580 & $2,456.5$ \\
\hline 17 & 3,000 & 0.1 & 10 & 0.549 & $2,313.8$ \\
\hline 18 & 3,500 & 0.1 & 10 & 0.587 & $2,668.1$ \\
\hline 19 & 4,000 & 0.1 & 12 & 0.322 & $3,133.4$ \\
\hline 20 & 3,500 & 0.1 & 12 & 0.232 & $2,774.5$ \\
\hline 21 & 3,500 & 0.08 & 12 & 0.259 & $2,257.3$ \\
\hline 22 & 3,500 & 0.06 & 12 & 0.273 & $1,722.3$ \\
\hline 23 & 3,000 & 0.08 & 12 & 0.368 & $1,953.8$ \\
\hline 24 & 4,000 & 0.06 & 12 & 0.246 & $1,953.8$ \\
\hline 25 & 3,000 & 0.06 & 12 & 0.214 & $1,487.3$ \\
\hline 26 & 4,000 & 0.08 & 12 & 0.210 & $2,555.0$ \\
\hline 27 & 3,000 & 0.1 & 12 & 0.206 & $2,406.9$ \\
\hline
\end{tabular}


Most of researchers have focused on the cutting parameters like speed, feed, and depth of cut while the tool-based parameters like tool diameters like tool diameter, tool path, helix angle, number of flutes, etc. have received scant attention. Therefore in the present work axial depth of cut and radial depth of cut were kept constant, and the effect of variation in tool diameter was considered. Literature reports that keeping the axial depth of cut at less than or equal to one-fourth of the final wall thickness results in less deformation (Modern Machine Shop, 2009). Also all the research works reported in the literature are based on straight, open walls. No work has been carried out on the thin wall machining of closed structures. It was therefore decided to use closed thin walled components for this study and record the impact of the process parameters on the geometrical accuracy.

A total of 27 experiments were carried out and the SR of the machined walls was measured at five different locations and the average value was calculated. Besides, the MRRs were also computed from the measured machining times. The collected experimental data are illustrated in Table 2 .

\section{Results and discussion}

Analysis of variance or 'ANOVA' is an analytical tool used to determine the significance of factors in an experiment by looking at the relationship between a response variable and a factor. In a simple factorial experiment comprising of three variables (A, B and C) at three levels (a levels of A, b levels of B and c levels of C), any observation can be described by the linear statistical model:

$$
\begin{aligned}
& Y_{i j k l}=\mu+\tau_{i}+\beta_{j}+\gamma_{k}+(\tau \beta)_{i j}+(\tau \gamma)_{i k}+(\beta \gamma)_{j k}+(\tau \beta \gamma)_{i j k}+\epsilon_{i j k l} \\
& \left\{\begin{array}{l}
i=1, \ldots, a \\
j=1, \ldots, b \\
k=1, \ldots, c \\
l=1, \ldots, n
\end{array}\right\}
\end{aligned}
$$

where $\mu$ is the overall mean effect, $\tau_{i}$ is the effect of $i^{\text {th }}$ level of factor $\mathrm{A}, \beta_{j}$ is the effect of $j^{\text {th }}$ level of factor B, $\gamma_{k}$ is the effect of the $k$-th level of factor $\mathrm{C},(\tau \beta)_{i j}$ is the effect of interaction between $\mathrm{A}$ and $\mathrm{B},(\tau \gamma)_{i k}$ is the effect of interaction between $\mathrm{A}$ and $\mathrm{C},(\beta \gamma)_{\mathrm{jk}}$ is the effect of interaction between $\mathrm{B}$ and $\mathrm{C},(\tau \beta \gamma)_{i j k}$ is the effect of interaction between $\mathrm{A}$, $\mathrm{B}, \mathrm{C}$ and $\epsilon_{i j k l}$ is a random error component having a normal distribution with zero mean.

The effect of a factor is the change in response produced by a change in level of the factor and is often called a main effect. ANOVA is used to test hypotheses about the main effects of the factors and their interactions. A hypothesis is a statement about some process parameter in an experiment whose validity is to be decided upon. The decision making procedure about the hypothesis is known as hypothesis testing. ANOVA tests these hypotheses by decomposing the total variability in the data into component parts and then comparing the various elements. The total variability is measured by the total sum of squares of the observations. From the total sum of squares the mean sum of squares is calculated as (Montgomery, 2014):

$$
M S_{i}=\frac{S S_{i}}{d}
$$


where, $M S_{i}$ is the mean square of treatment $i, S S_{i}$ is the sum of squares of treatment $i$ and $d$ is the degree of freedom and is calculate as shown in Table 3. Next, the $F_{o}$ value is calculated as

$$
F_{0}=\frac{M S_{i}}{M S_{e}}
$$

where $M E_{e}$ is the mean square error. After the $F_{o}$ values are calculated, the $p$-values are calculated using R programming language.

Table 3 Levels of experimental process parameters and degrees of freedom

\begin{tabular}{lccccc}
\hline Parameter & Code & Level 1 & Level 2 & Level 3 & Degrees of freedom \\
\hline Feed (mm/tooth) & $\mathrm{A}$ & 0.06 & 0.08 & 0.1 & $(\mathrm{a}-1)=2$ \\
Speed (rpm) & $\mathrm{B}$ & 3,000 & 3,500 & 4,000 & $(\mathrm{~b}-1)=2$ \\
Tool diameter (mm) & $\mathrm{C}$ & 8 & 10 & 12 & $(\mathrm{c}-1)=2$ \\
Interaction (feed-speed) & $\mathrm{AB}$ & - & - & - & $(\mathrm{a}-1)(\mathrm{b}-1)=4$ \\
Interaction (feed-tool diameter) & $\mathrm{AC}$ & - & - & - & $(\mathrm{a}-1)(\mathrm{c}-1)=4$ \\
Interaction (speed- tool diameter) & $\mathrm{BC}$ & - & - & - & $(\mathrm{b}-1)(\mathrm{c}-1)=4$ \\
Interaction (three factor) & $\mathrm{ABC}$ & - & - & - & $(\mathrm{a}-1)(\mathrm{b}-1)(\mathrm{c}-1)=8$ \\
Error & $\mathrm{E}$ & - & - & - & $\mathrm{abc}(\mathrm{n}-1)=27$ \\
Total & $\mathrm{T}$ & - & - & - & $(\mathrm{abcn}-1)=53$ \\
\hline
\end{tabular}

\subsection{ANOVA for SR}

The ANOVA for SR is illustrated in Table 4.

Table 4 ANOVA for SR

\begin{tabular}{lccccc}
\hline \multirow{2}{*}{ Source of variation } & $\begin{array}{c}\text { Sum of squares } \\
\text { SS }\end{array}$ & $\begin{array}{c}\text { DOF } \\
\text { Feed (A) }\end{array}$ & $\begin{array}{c}\text { Mean square } \\
M S_{i}\end{array}$ & $F_{o}$ & p-value \\
\hline Speed (B) & 0.2062 & 2 & 0.1031 & 1.1385 & 0.3350 \\
Tool diameter (C) & 0.0083 & 2 & 0.0042 & 0.0461 & 0.9551 \\
AB & 23.8093 & 2 & 11.9046 & 131.4618 & $1.21 \mathrm{E}-14$ \\
AC & 0.0916 & 4 & 0.0229 & 0.2528 & 0.9053 \\
BC & 0.3237 & 4 & 0.0809 & 0.8938 & 0.4811 \\
ABC & 0.1777 & 4 & 0.0444 & 0.4905 & 0.7430 \\
Error & 0.9953 & 8 & 0.1244 & 1.3739 & 0.2520 \\
Total & 2.4450 & 27 & 0.0906 & 1.0000 & \\
\hline
\end{tabular}

Now the critical values of $F$ for $95 \%$ confidence (i.e. $\alpha=0.05$ ) are:

\begin{tabular}{ll}
\hline DOF & $F_{\text {critical }}$ \\
\hline 2,27 & 3.35 \\
4,27 & 2.73 \\
8,27 & 2.31 \\
\hline
\end{tabular}


From the above analysis the following observations are made:

- $\quad$ The tool diameter $(\mathrm{C})$ significantly affects SR since the $F_{o}$ value, 131.4618 , is significantly higher than the critical value of 3.35 . Besides, the p-value $1.21 \times 10^{-14}$ is also significantly lower than $\alpha$. Thus the null hypothesis, $H_{o}$, is rejected for $\mathrm{C}$.

- There is no interaction between the factors for SR as the $F_{o}$ values are below the corresponding $F_{\text {critical }}$ values. This means that the change in SR due to change in the level of one process parameter is independent of the other process parameters.

- Tool diameter is the most significant parameter for both deformation and SR. The order of significance is:

\section{Tool diameter}

Figure 3 shows the relative significance of the process parameters for SR. From the study it can be seen that the most significant parameter is the tool diameter (C).

Figure 3 Percentage contributions of process parameters to SR

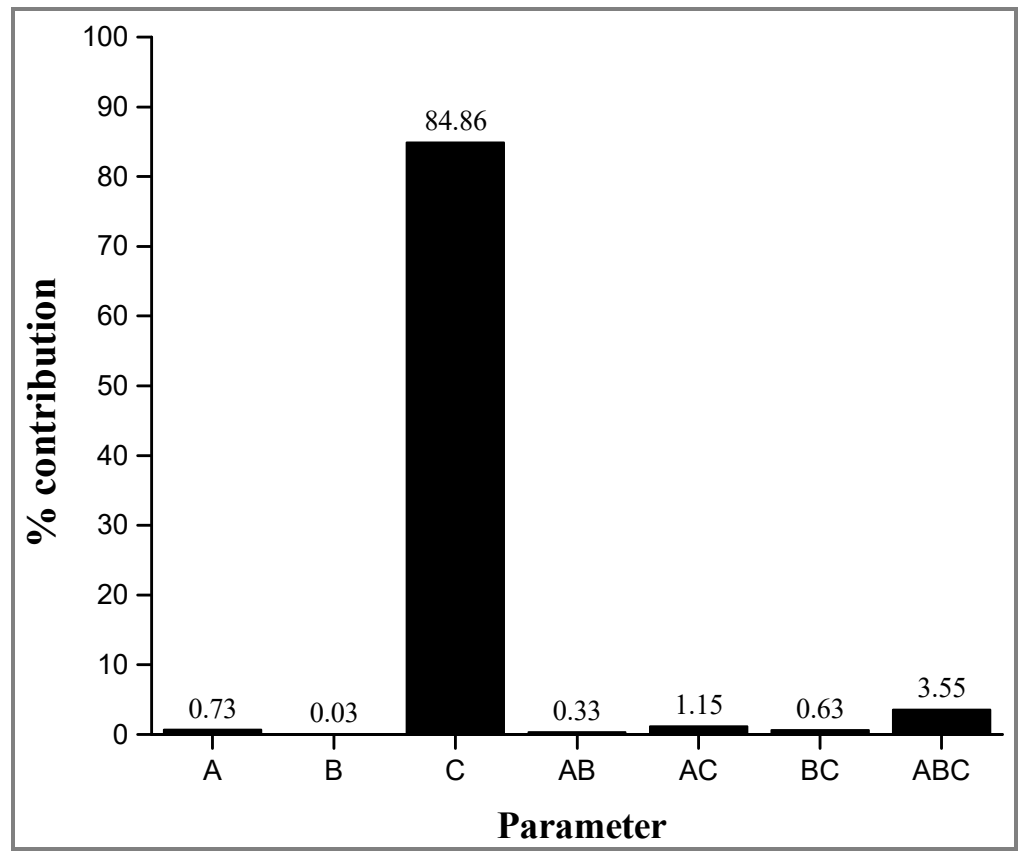

\subsection{Effect of process parameters on $S R$}

From Figure 4(a) it can be seen that the $R_{a}$ value increases with increase in feed per tooth from 0.06 to $0.08 \mathrm{~mm} /$ tooth. But there is a slight decrease in $R_{a}$ with further increase in feed from 0.08 to 0.1 . This observation can be explained on the basis of equation (Juneja and Seth, 2003), 


$$
R_{a}=\frac{8 f_{t}^{2}}{9 \sqrt{3} D}
$$

where $R_{a}$ is the average $\mathrm{SR}, f_{t}$ is the feed per tooth and $D$ is the tool diameter.

Figure 4 Main effect plots for SR, (a) main effect of feed (b) main effect of speed (c) main effect of tool diameter

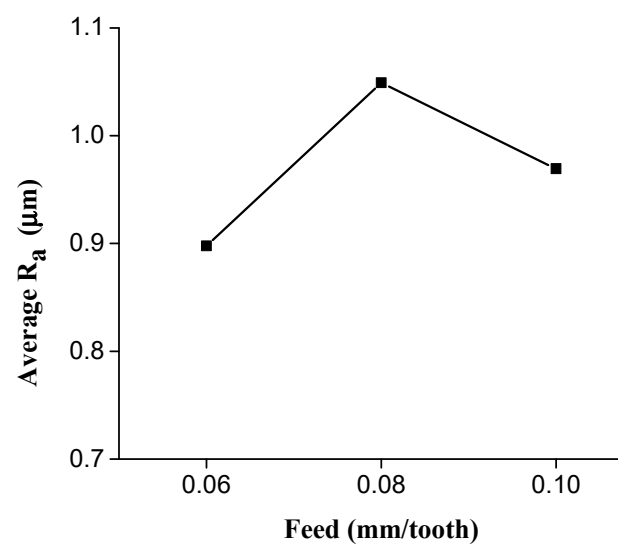

(a)

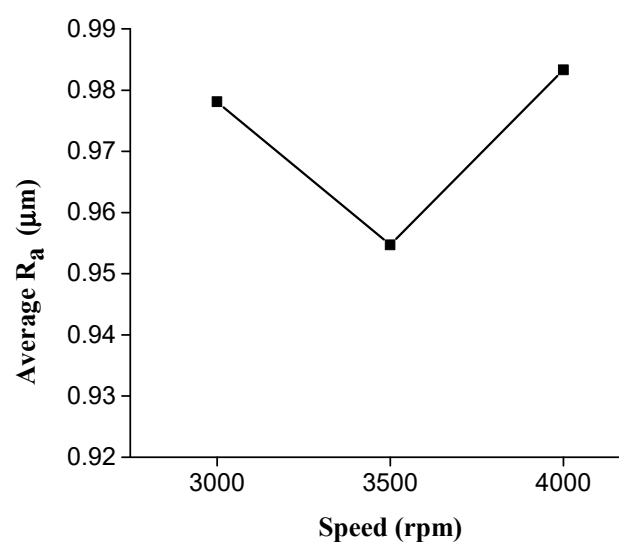

(b)

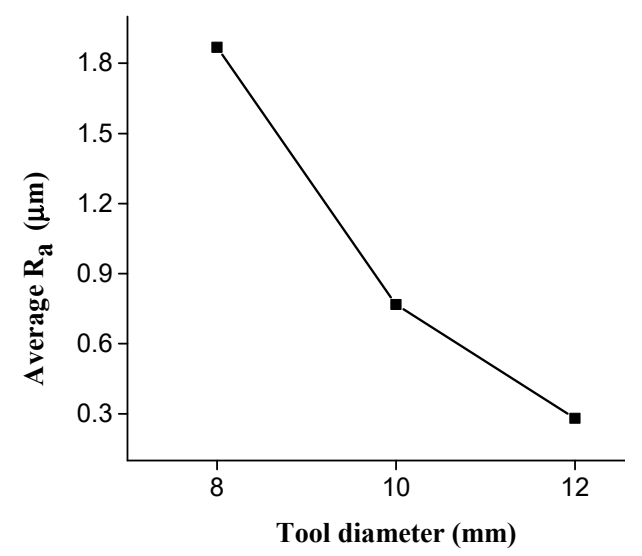

(c)

It implies that the $R_{a}$ is directly proportinal to the square of the feed per tooth. Thus the $R_{a}$ value should increase with increase in feed per tooth, which is the global trend observed in the experimental data. However, there is a decrease in $R_{a}$ with further increase in feed.

Figure 4(b) shows the effect of speed on SR. It is observed that the $R_{a}$ value decreases with increase in speed from 3,000 rpm to 3,500 rpm. However, with the increase in speed from $3,500 \mathrm{rpm}$ to $4,000 \mathrm{rpm}$, the $R_{a}$ value increases. At low speeds the cutting forces are high and there is a possibility of the formation of built-up edges which deteriorates the surface finish. At higher speeds, the cutting forces and the tendency to form built-up edge decreases due to increase in cutting temperature and the decrease in stress at the rake 
face. Thus the surface finish improves with increasing speed. The effect of spindle speed on the SR is a bit complicated as this invloves the machine paramters as well. At a particular spindle speed the frequency of the cutter striking the workpiece may become equal to the natural frequency of the workpiece. This lead to a condition known as resonance which is characterised by large amplitudes of deflection resulting in vibration and chatter. This leads to a poor surface finish, i.e., a high value so $\mathrm{R}_{\mathrm{a}}$. From Figure 4(b) it can be seen that at first the $R_{a}$ value decreases with increase in the spindle speed but then increases with further increase in speed. This is due to the fact that there is considerable amount of chatter due to vibrations near the 4,000 rpm mark for the machine used for the experiments which resulted in high $\mathrm{R}_{\mathrm{a}}$ values.

From Figure 4(c), it is observed that the $R_{a}$ value decreases globally with increase in tool diameter. The variation in the $R_{a}$ values can be explained by means of the expression for SR given by equation (5) which states that $R_{a}$ is inversely proportional to the tool diameter. With an increase in tool diameter for the same speed and feed, the height of irregularities on the surface reduces due to which the $R_{a}$ value decreases as shown in Figure 5 .

Figure 5 Effect of increase in tool diameter on roughness height
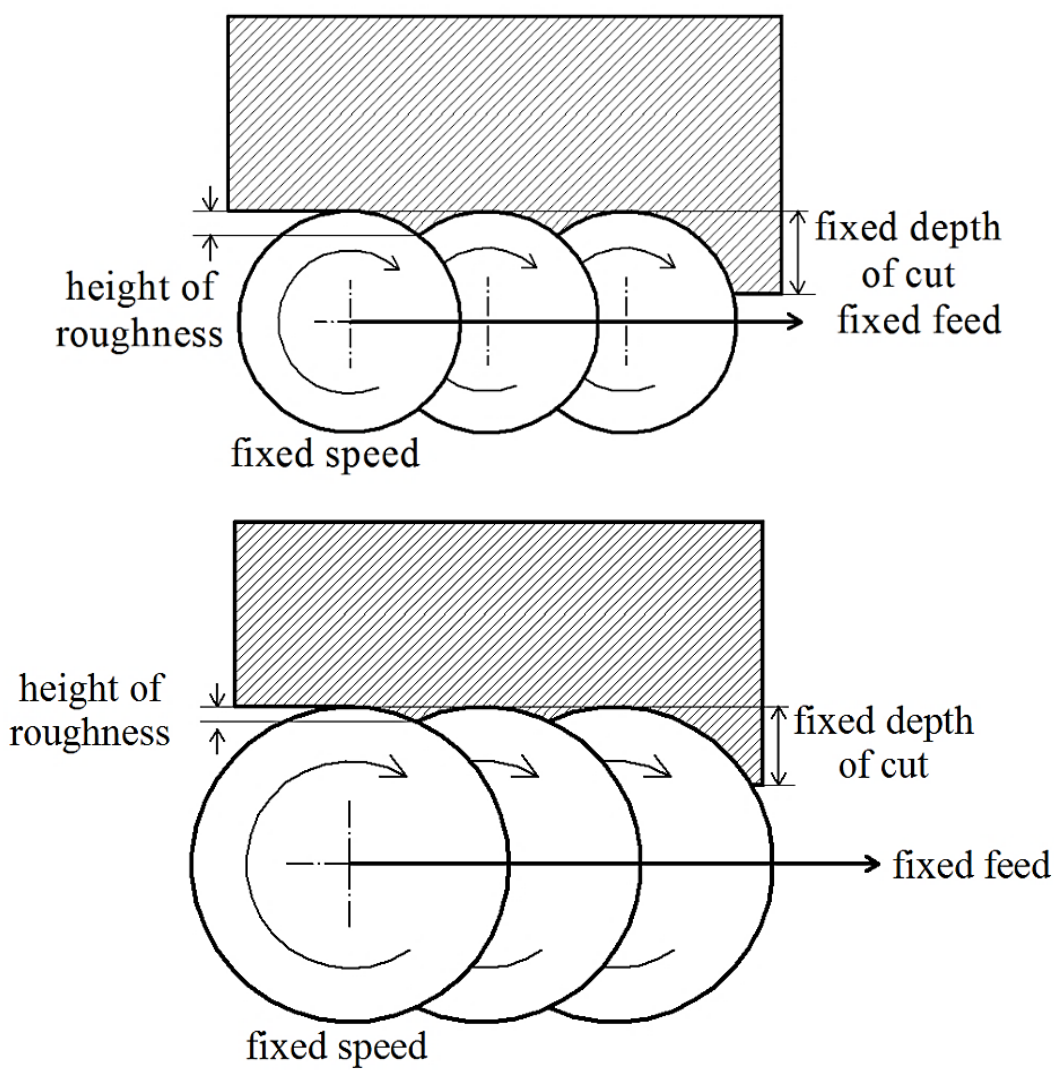
The interaction plots for $R_{a}$ are shown in Figure 6. From the interaction plots it can be seen that there is interaction between feed and speed but no such interaction is indicated from the ANOVA. It can be seen that the graphs have nearly equal slopes and thus it may be concluded that there is no interaction between feed and speed. The same can be concluded for the others where the graphs are nearly parallel with very little difference in slopes.

Figure 6 Interaction plots for SR (see online version for colours)

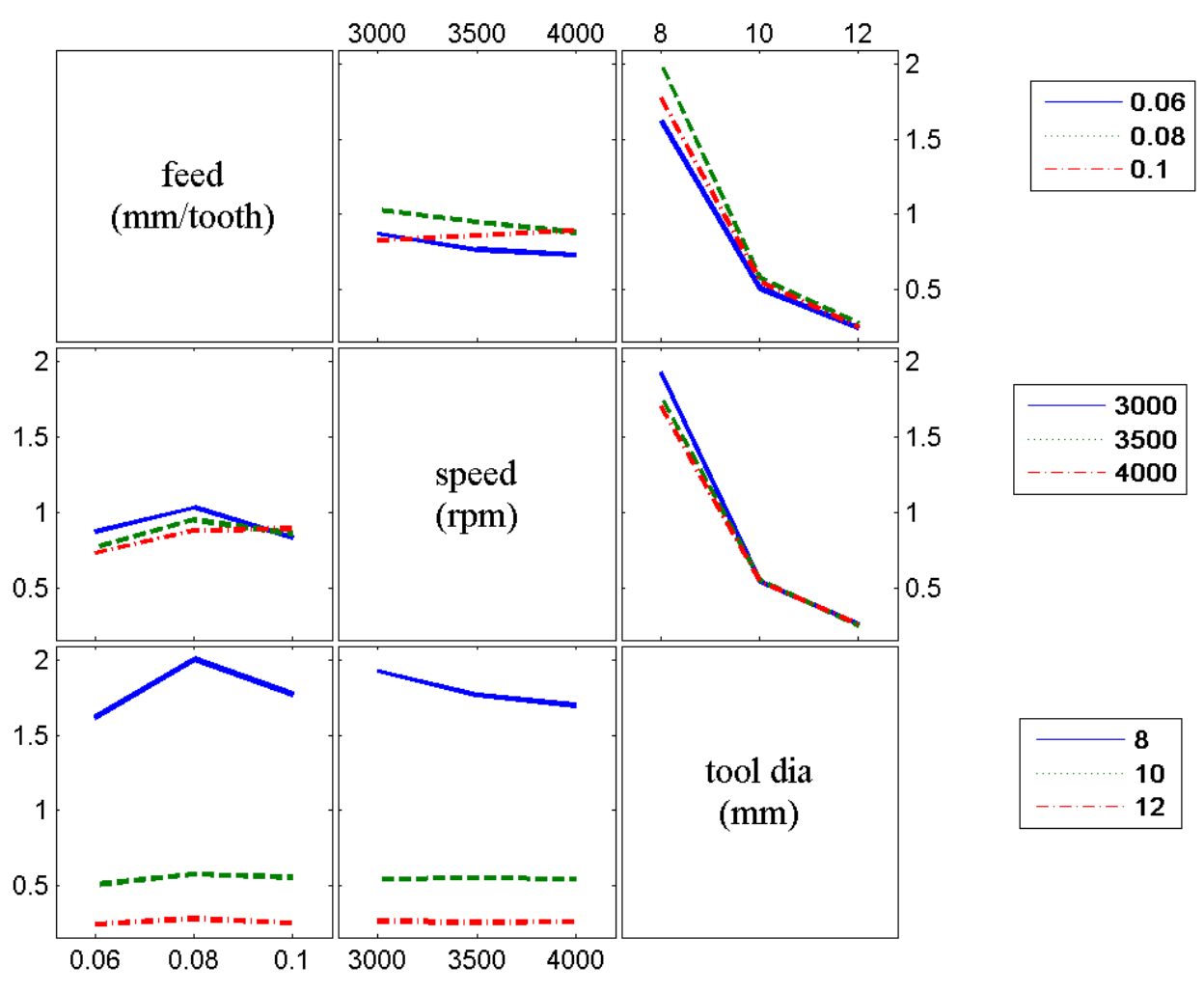

\subsection{Effect of process parameters on MRR}

Figure 7 shows the main effects plots for MRR. MRR for milling can be expressed as,

$$
M R R=\alpha_{p} \times \alpha_{e} \times f_{t} \times N \times z
$$

where $a_{p}$ is the axial depth of cut, $a_{e}$ is the radial depth of cut, $f_{t}$ is the feed per tooth, $N$ is the spindle speed and $z$ is the number of tooth of the milling cutter. 
Figure 7 Main effect plots for MRR, (a) main effect of feed (b) main effect of speed (c) main effect of tool diameter

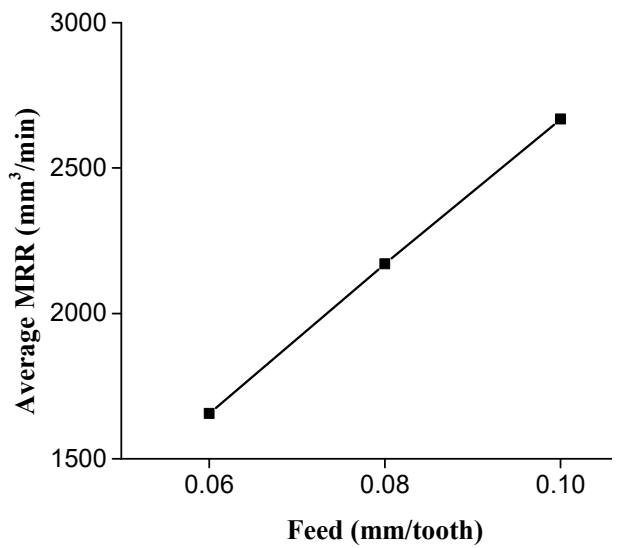

(a)

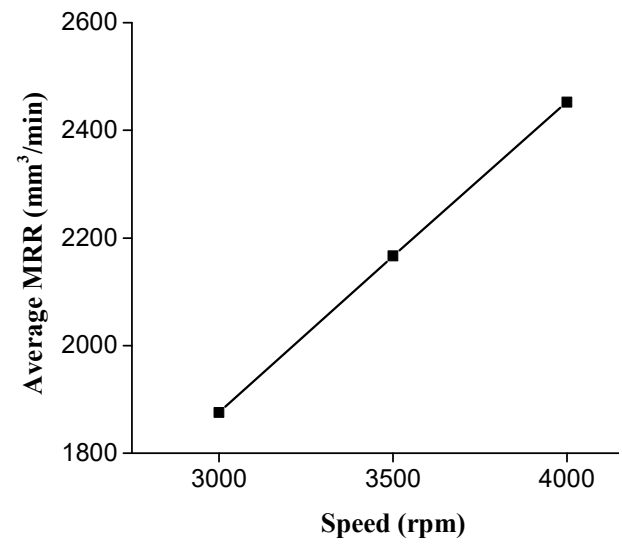

(b)

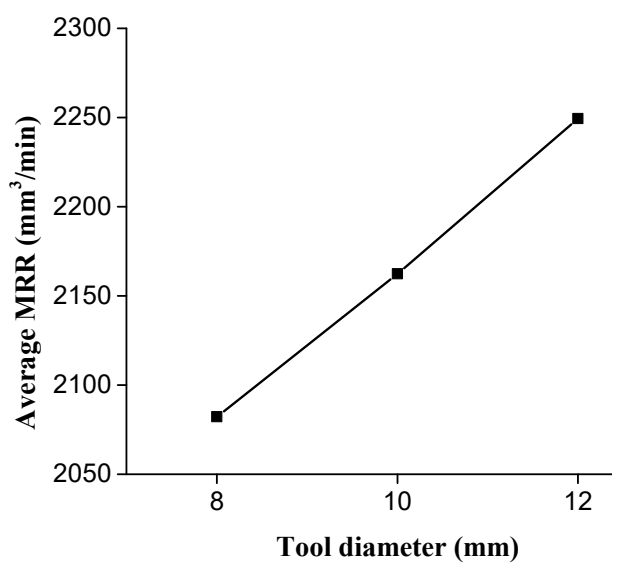

(c)

It can be seen from Figure 7(a) that the MRR increases with increase in feed. This is because the cutter traverses more distance with higher feed rates in the same time, thereby increasing the MRR. Besides from equation (6) it is evident that the MRR is directly proportional to the feed per tooth. Figure $7(b)$ shows that the MRR is higher for higher spindle speed. For the same feed and tool diameter, when the speed increases, there is an increase in the number of contacts made by the cutter with the workpiece. This increases the number of cutting motions per pass which results in more amount of material removal. Therefore an increase in spindle speed is accompanied by a corresponding increase in the MRR. The same can be concluded from equation (6), which states that the MRR is directly proportional to the spindle speed.

From Figure 7(c), it can be seen that the MRR increases with an increase in tool diameter. For the same feed per tooth and spindle speed, an increase in tool diameter will cause more of the tool going inside the workpiece, thereby increasing the MRR. 
Figure 8 Interaction plots for MRR (see online version for colours)

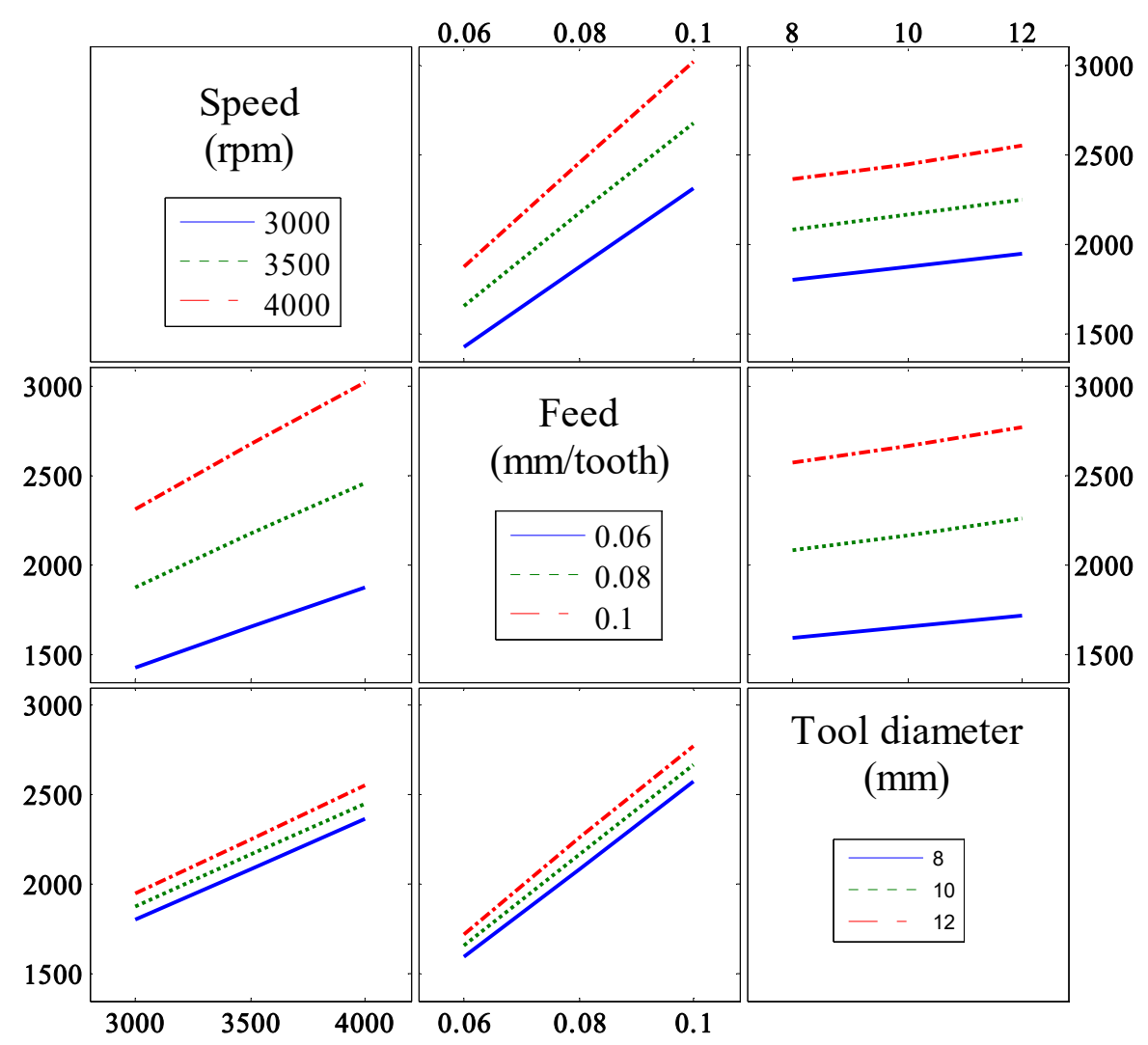

Figure 8 shows the interaction plots for MRR. From the plots it is seen that the graphs are parallel to each other which indicates that there is no interaction between the process parameters. This means that the variation of MRR with the change in level of any one of the process parameters is independent of the other parameters.

\section{Optimisation of process parameters using firefly algorithm}

\subsection{Firefly algorithm}

Optimisation can be classified in various ways. One of the simplest ways to classify optimisation is to categorise them in two types: deterministic and stochastic. Deterministic algorithms search for optimum point in a predefined path. On the contrary stochastic algorithms include randomisation which increases the area of search thus making them more suitable for global optimisation. While most of the conventional algorithms are deterministic in nature, the nature inspired algorithms are stochastic. In the modern scenario there exist a good number of nature inspired algorithms. Among these algorithms particle swarm optimisation, cuckoo search and firefly algorithms are well established into the area of optimisation as they are proven to be very efficient. With respect to various nature inspired algorithms, firefly algorithm is one of the most recently 
developed optimisation technique. Yang (2009) carried out a systematic study comparing firefly algorithm, particle swarm optimisation and genetic algorithm to find out the best technique with respect to efficiency and effectiveness. The findings reveal that the firefly algorithm is superior to both particle swarm optimisation and genetic algorithm in terms of both efficiency and success rate. Gandomi et al. (2011) employed firefly algorithm to solve mixed variable structural optimisation problem. The optimisation results showed that firefly algorithm is more efficient than particle swarm optimisation, genetic algorithm, simulated annealing and hunting search. Apostolopoulos and Vlachos (2010) used firefly algorithm to minimise fuel cost and emission of generating units in a power plant. The results depicted the accuracy and high success rate of the firefly algorithm in searching for global optimum. Senthilnath et al. (2011) used firefly algorithm for clustering and the results were compared with the particle swarm optimisation, artificial bee colony and other famous algorithms. This comparison favoured firefly algorithm to be more worthy and efficient for global optimisation. Based on all the above studies it was therefore decided to use firefly algorithm for optimisation of thin wall machining parameters.

Firefly algorithm is a kind of swarm optimisation technique (Yang, 2009). Swarm intelligence is a form of artificial intelligence that is inspired from the collective behaviour of animals and insects alike. The collective system is self-organised and decentralised consisting of simple agents that organise themselves using neighbourhood interactions. The individual agents are relatively unsophisticated individuals but they exhibit coordinated behaviour that directs the swarm towards their desired goals.

Firefly algorithm is such a stochastic, meta-heuristic algorithm which can be used to solve a wide variety of optimisation problems and is inspired by the self-organised behaviour of the fireflies. Fireflies are known for their characteristic short and rhythmic flashing lights which are produced by a process of bioluminescence. Although, the true functions of the flashes are still debated, two fundamental functions, which are generally accepted, are to attract mating partners and to attract potential prey besides warning other predators of their bitter taste. In the firefly algorithm, the flashing light, which is inversely proportional to the square of the distance from the source, is formulated in such a way that it is associated with the objective function to be optimised. For simplicity of analysis the following assumptions were made (Iztok et al., 2013):

- All fireflies are unisex, so one firefly will be attracted to other fireflies regardless of their sex.

- Attractiveness is proportional to a firefly's brightness. Thus for any two flashing fireflies, the dimmer one will move toward the brighter one. The attractiveness is proportional to the brightness, both of which decrease as their distance increases. If there is no brighter one than a particular firefly, it will move randomly.

- The brightness of a firefly is affected or determined by the landscape of the objective function.

On the basis of the rules stated above, the firefly algorithm can be summarised by the following pseudo-code (Iztok et al., 2013): 


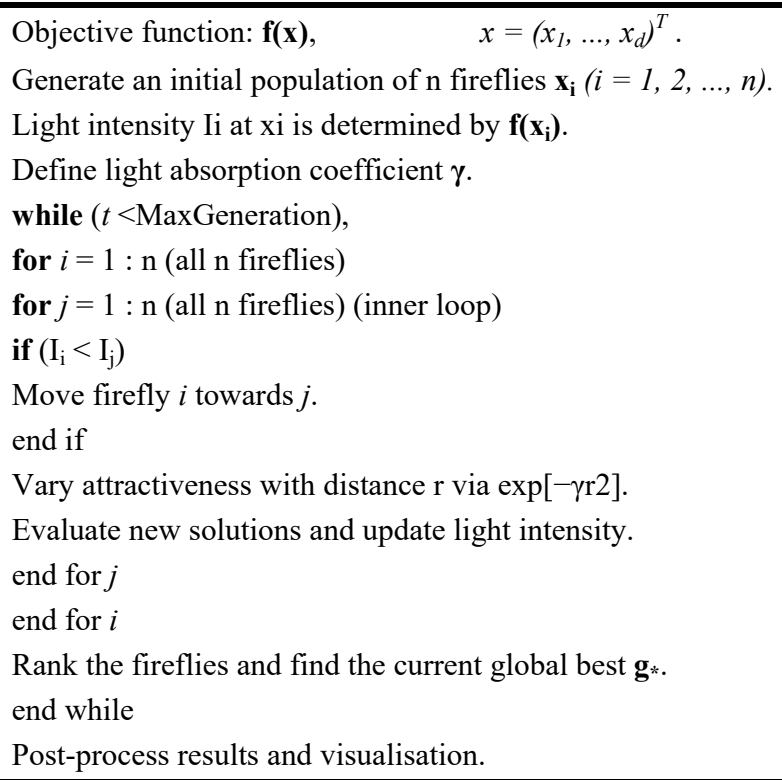

Formulation of firefly algorithm involves two important aspects: variation of light intensity and formulation of attractiveness. It is assumed that the attractiveness of a firefly is a function of its brightness which in turn is a determined by the objective function.

The light intensity $I$ of a firefly relative to another firefly at a distance $r$ from it is given by:

$$
I(r)=I_{0} e^{-\gamma r^{2}}
$$

where $I_{0}$ is the intensity at the source $(r=0)$ and $\gamma$ is the light absorption coefficient.

Similarly, the attractiveness $(\beta)$ of a firefly, which is proportional to the light intensity as seen by the other fireflies, can be formulated as follows:

$$
\beta(r)=\beta_{0} e^{-\gamma r^{2}}
$$

where $\beta_{0}$ is the attractiveness at $r=0$.

The light intensity and attractiveness though appear to be similar in some aspects, there is a major difference between them. The light intensity is an absolute measure of the light emitted by a firefly whereas the attractiveness is a relative measure of the light that is seen and judged by any other firefly. Moreover, the distance between any two fireflies $x_{i}$ and $x_{j}$ is expressed as follows:

$$
r_{i j}=\left\|x_{i}-x_{j}\right\|=\sqrt{\sum_{k=1}^{d}\left(x_{i k}-x_{j k}\right)^{2}}
$$

where $x_{i, k}$ is the $k^{\text {th }}$ component of the spatial coordinate $x_{i}$ of $i^{\text {th }}$ firefly.

The movement of a firefly $i$ attracted to another, brighter firefly $j$ is determined by:

$$
x_{i}=x_{i}+\beta_{0} e^{-\gamma r_{i j}^{2}}\left(x_{j}-x_{i}\right)+\alpha \varepsilon_{i}
$$


where $\alpha \in[0,1]$ is the randomisation parameter and $\epsilon_{i}$ is a random number drawn from Gaussian distribution. The parameter $\gamma$ has a crucial impact on the convergence speed whose settings depends on the optimisation problem. Its value varies between 0.1 and 10 .

Figure 9 Variation of MRR with process parameters

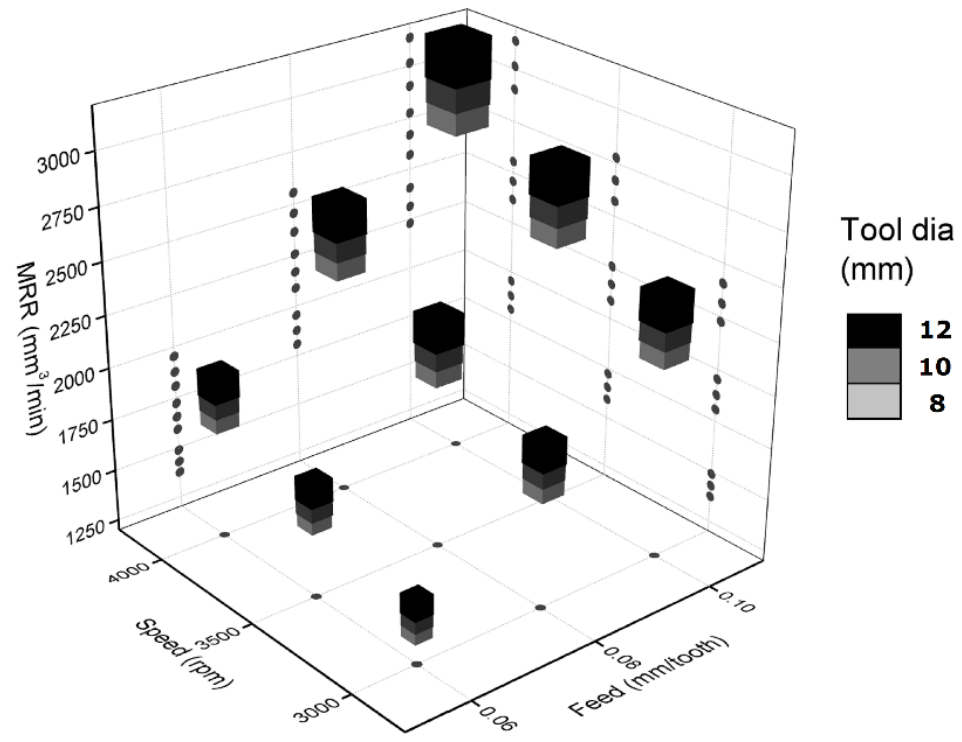

Figure 10 Variation of SR with process parameters

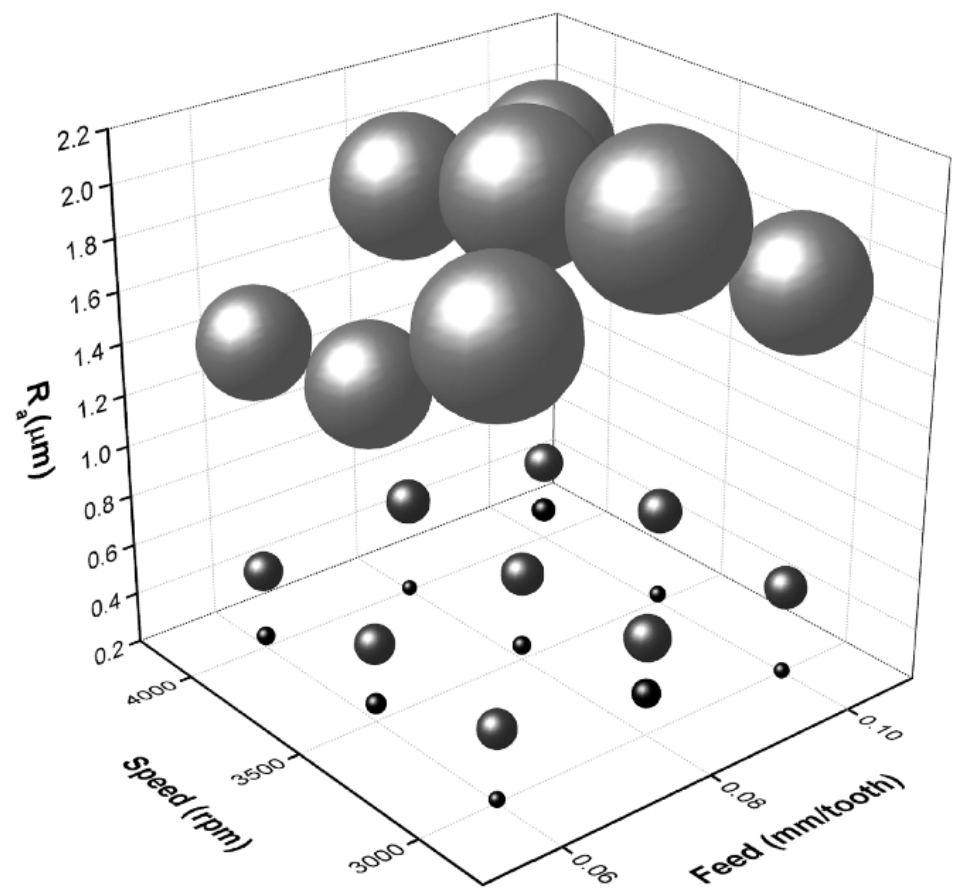


Thin wall machining is used to produce parts from solid metal/alloy blocks by removing up to $95 \%$ of the material. Thus, the overall productivity of the process depends mostly on the MRR. To achieve higher MRRs, higher feed, speed and depth of cut along with larger tool diameter are essential. But the use of higher levels of these parameters may be detrimental to overall product quality in the form of geometrical inaccuracy and poor surface finish. The variation of SR and MRR as recorded during the experiments is shown in Figures 9 and 10. It can be seen that the MRR increases with increase in tool diameter while the SR decreases. Similarly, high speed and feed lead to high MRR but poor surface finish.

Thus, the objective function can be composed of a linear combination of SR and MRR. For maximising productivity, MRR must be maximised while keeping SR at the minimum. To achieve this, a single objective function is developed from the individual regression equations for SR [equation (11)] and MRR [equation (12)]. The regression equations are developed in MATLAB using the functions polyfitn and polyvaln developed by John D'Errico which is freely available at MATLAB central file exchange (MATLAB Central, 2012).

$$
\begin{aligned}
S R= & 17.97441+(44.17106) A-\left(1.44209 \times 10^{-3}\right) B-(2.92148) C \\
& +\left(5.13990 \times 10^{-3}\right) A B+\left(5.6089510^{-5}\right) B C-(0.90572) A C \\
& -(320.90783) A^{2}+\left(5.61171 \times 10^{-8}\right) B^{2}+(0.12065) C^{2} \\
M R R & =-2,297.15940+(25,320.05785) \mathrm{A}+(0.57670) \mathrm{B}+(41.77596) \mathrm{C}
\end{aligned}
$$

where $A=$ feed, $B=$ speed and $C=$ tool diameter.

For the regression equations the following parameters were recorded:

Table 5 Details of regression analysis

\begin{tabular}{lcc}
\hline Parameter & Surface roughness & Material removal rate \\
\hline R-squared & 0.9831 & 0.9915 \\
Adjusted R-squared & 0.9741 & 0.9904 \\
Predicted R-squared & 0.9553 & 0.9871 \\
Adequate precision & 26.965 & 94.544 \\
\hline
\end{tabular}

where

- R-squared: a measure of the amount of variation around the mean explained by the model.

- $\quad$ Adjusted R-squared: a measure of the amount of variation around the mean explained by the model adjusted for the number of terms in the model.

- $\quad$ Predicted R-squared: a measure of the amount of variation in new data explained by the model.

- Adequate precision: it is a signal to noise ratio. It compares the range of predicted values at the design points to the average prediction error.

Now, we have to formulate a single objective function from equations (11) and (12). The overall objective function will be of the form: 


$$
y=w_{1} \times M R R-w_{2} \times S R
$$

where $w_{1}$ and $w_{2}$ are the weights to be decided upon. These weights indicate the precedence or bias toward either of the parameters. For our case, we are supposed to get maximum MRR without compromising the surface quality. Therefore a value of 0.5 was chosen for both $w_{1}$ and $w_{2}$. It is to be noted that if SR is more important to the experimenter then more weightage should be put on $w_{2}$ and vice-versa. Since the SR is to be minimised, its weight $w_{2}$ is preceded with a minus sign.

The next and the most important step is to decide upon the methodology to be used for the optimisation. There are a number of optimisation techniques prevalent in literature including genetic algorithm-based techniques and swarm optimisation techniques like particle swarm optimisation, firefly algorithm, cuckoo search algorithm, etc. Among all these techniques, firefly algorithm was chosen as the preferred optimisation technique based on the findings of Yang (2009).

The code for firefly algorithm is open source and freely available from http://www.mathworks.in. The MATLAB code is slightly modified to include the objective function given by equation (13) and also to include provision for data extraction and plotting. The results obtained after running the optimisation code are as follows:

Table 6 Optimised process parameters

\begin{tabular}{lc}
\hline Parameter & Optimised value \\
\hline Feed $(\mathrm{mm} /$ tooth) & 0.1 \\
Speed $(\mathrm{rpm})$ & 3839 \\
Tool diameter $(\mathrm{mm})$ & 10 \\
\hline
\end{tabular}

By putting these values in equations (11) and (12) the obtained optimum values of SR and MRR are shown in Table 7. It can be that the optimised process parameters yield considerably good results. The MRR obtained is $32.42 \%$ higher than the average value whereas the SR value obtained is about $37.25 \%$ lower than the average value.

Table 7 Productivity and quality improvement using optimised results

\begin{tabular}{lccc}
\hline Parameter & Optimised value & Average values & \% improvement \\
\hline $\mathrm{MRR}\left(\mathrm{mm}^{3} / \mathrm{min}\right)$ & 2866.557 & 2164.643 & 32.42 \\
$\mathrm{SR}(\mu \mathrm{m})$ & 0.5443 & 0.86746 & 37.25 \\
\hline
\end{tabular}

\section{Confirmation experiment}

A confirmation experiment was carried out to validate the optimisation results. The experiment was carried out using the optimised set of process parameters derived using firefly algorithm given in Table 7 . After the machining process, the resulting surface finish of the component is measured at 5 different locations and the average $R_{a}$ value is measured. Similarly the MRR is also calculated from the measured machining times. These values are then compared with the values obtained by putting the optimised values of the process parameters in the regression equations for $R_{a}$ and $M R R$ given by equations (11) and (12) respectively. It is found that the prediction results are quite 
accurate with an average absolute error of $1.27 \%$ for SR and $1.03 \%$ for MRR as shown in Table 8 .

Table 8 Comparison between predicted and experimental results

\begin{tabular}{lccc}
\hline Outputs & Optimised & Experimental & \% error \\
\hline MRR $\left(\mathrm{mm}^{3} / \mathrm{min}\right)$ & $2,866.557$ & $2,903.681$ & 1.27 \\
$\mathrm{SR}(\mu \mathrm{m})$ & 0.5443 & 0.550 & 1.03 \\
\hline
\end{tabular}

\section{Conclusions}

In this study the effects of process parameters on the SR and MRR obtained during thin wall machining of monolithic closed geometrical components are presented. Analysis of variation is carried out on the experimental data and the interaction among the variables is studied. From the study it is found that tool diameter is the most important parameter for SR. Further, the process parameters are optimised using firefly algorithm and it is found that the optimised set of process parameters results in an improvement of $32.42 \%$ and $37.25 \%$ over the average values of MRR and SR respectively. The results are validated by conducting a confirmation experiment which indicates an error of $1.27 \%$ and $1.03 \%$ between predicted and experimental results of MRR and SR respectively.

\section{Acknowledgements}

This work was supported by the Science and Engineering Research Board (SERB), Department of Science and Technology, Government of India (Grant number SR-S3-MERC-0115-2012).

\section{References}

Apostolopoulos, T. and Vlachos, A. (2010) 'Application of the firefly algorithm for solving the economic emissions load dispatch problem', International Journal of Combinatorics, Vol. 2011, Article ID 523806, 23pp, doi: 10.1155/2011/523806.

Gandomi, A.H., Yang, X-S. and Alavi, A.H. (2011) 'Mixed variable structural optimization using firefly algorithm', Computers \& Structures, Vol. 89, No. 23, pp.2325-2336.

Hayajneh, M.T., Tahat, M.S. and Bluhm, J. (2007) 'A study of the effects of machining parameters on the surface roughness in the end-milling process', Jordan Journal of Mechanical and Industrial Engineering, Vol. 1, No. 1, pp.1-5.

Hossain, M.S.J. and Ahmad, N. (2012) 'Artificial intelligence based surface roughness prediction modeling for three dimensional end milling', International Journal of Advanced Science and Technology, August, Vol. 45, pp.1-18.

Juneja, B.L. and Seth, N. (2003) Fundamentals of Metal Cutting and Machine Tools, New Age International, New Delhi, India.

Kennedy, B. and Earls, A.R. (2007) 'Wall smart: thin-wall milling isn't for the faint of heart, but techniques exist for performing it efficiently', Cutting Tool Engineering, Vol. 59, No. 2.

MATLAB Central (2012) [online] http://www.mathworks.com/matlabcentral/fileexchange/34765polyfitn (accessed February 2015). 
Michalik, P., Zajac, J., Hatala, M., Mital, D. and Fecova, V. (2014) 'Monitoring surface roughness of thin-walled components from steel C45 machining down and up milling', Measurement, December, Vol. 58, pp.416-428.

Modern Machine Shop (2009) [online] http://www.mmsonline.com/articles/how-to-machineaircraft-titanium-the-8-to-1-rule-for-finishing-walls-and-ribs. (accessed January 2015).

Montgomery, D.C. (2014) Design and Analysis of Experiments, John Wiley \& Sons, New Delhi, India.

Seguy, S., Dessein, G. and Arnaud, L. (2008) 'Surface roughness variation of thin wall milling, related to modal interactions', International Journal of Machine Tools and Manufacture, Vol. 48, No. 3, pp.261-274.

Senthilnath, J., Omkar, S.N. and Mani, V. (2011) 'Clustering using firefly algorithm: performance study', Swarm and Evolutionary Computation, Vol. 1, No. 3, pp.164-171.

Shamsuddin, K.A., Ab-Kadir, A.R. and Osman, M.H. (2013) 'A comparison of milling cutting path strategies for thin-walled aluminum alloys fabrication', The International Journal of Engineering and Science, Vol. 2, No. 3, pp.01-08.

Thin Wall Machining (2015) [online] http://www.makino.com/about/news/thin-wallmachining/171 (accessed February 2015).

Wang, W., Kweon, S.H. and Yang, S.H. (2005) 'A study on roughness of the micro-end-milled surface produced by a miniatured machine tool', Journal of Materials Processing Technology, 15 May, Vol. 162, pp.702-708.

Yang, G. (1980) Elastic and Plastic Mechanics, People Education Published Inc., PRC.

Yang, X.S. (2009) Firefly Algorithms for Multimodal Optimization. Stochastic Algorithms: Foundations and Applications, Springer Berlin Heidelberg, Heidelberg, Germany, pp.169-178.

Iztok, F., Yang X.S. and Brest, J. (2013) 'A comprehensive review of firefly algorithms', Swarm and Evolutionary Computation, December, Vol. 13, pp.34-46. 